In Brian Bruya (ed.), Effortless Attention: A New Perspective in the Cognitive Science of Attention and Action. Cambridge, MA: MIT Press, 2010.

\title{
11 Apertures, Draw, and Syntax: Remodeling Attention
}

\section{Brian Bruya}

Because psychological studies of attention and cognition are most commonly performed within the strict confines of the laboratory or take cognitively impaired patients as subjects (Parasuraman 1998b; Pashler 1998; Posner 2004; Underwood 1993), it is difficult to be sure that resultant models of attention adequately account for the phenomenon of effortless attention. The problem is not only that effortless attention is resistant to laboratory study (but see Moller, Meier, and Wall, chapter 9, this volume). A further issue is that because the laboratory is the most common way to approach attention, models resulting from such studies are naturally the most widely propagated, these models naturally tend to be biased toward features of attention most amenable to laboratory study, and these models by their implications set the agenda for future study that leads back to the laboratory. In this self-reinforcing system, features of attention not amenable to laboratory study are naturally neglected by researchers. Being that they are neglected, one can surmise that they are not adequately accounted for in current models, and such models, therefore, fail to indicate potentially important areas for future study. In this chapter, I will suggest an alternative model of attention as a heuristic for opening paths to further profitable research. The features of attention emphasized in this model are not new, but the synthesis is novel and sheds some light on issues relevant to the topic of effortless attention.

I begin with the five following observations:

1. One naturally pays attention to a task of current interest.

2. There are (at least) two distinct modes of attention—selective and diffuse.

3. Attention is a constantly shifting avenue for the assimilation of information.

4. Information is not forced in from outside but is captured through internal sensitization.

5. Human information processing is fundamentally syntactic.

Combining these five observations yields an explanatory model of attention that is not only consistent with the data from the many studies on attention in recent decades but also allows us to investigate the neglected phenomenon of effortless 


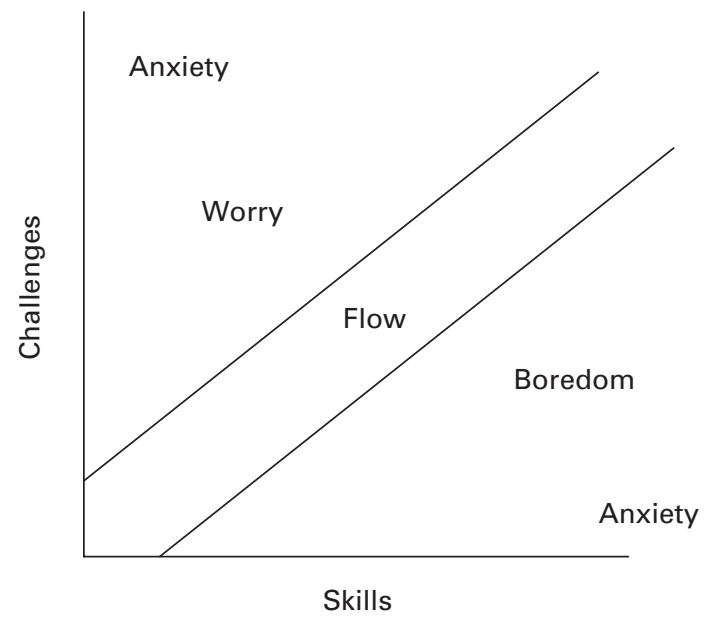

Figure 11.1

Psychological states related to challenge and skill balance (Csikszentmihalyi and Csikszentmihalyi).

attention. The model relies on the notions of apertures, draw, and syntax (ADS, for short) and will be explicated by addressing each of the above observations in turn. In the final part of the chapter, I shall explore how the model expands our understanding of effortless attention.

\section{Interest and Effortless Attention}

Csikszentmihalyi and Csikszentmihalyi $(1988,49)$ postulated a flow channel that accounts for effortless attention and action in terms of a balance between challenges and skills (see figure 11.1):

Massimini and Carli (Csikszentmihalyi 1997, 31) revised this model, specifying further states related to the imbalance of challenges and skills (see figure 11.2).

Under these interpretations, the states of arousal, flow, control, and relaxation are all positive affective states. This positive affect partially explains natural human motivation for pursuing and achieving activities that engender flow. However, positive affect alone does not tell the whole story.

Under normal circumstances, attention is most easily maintained if one is actively interested, so an obvious place to turn is the notion of interest. Paul Silvia (2006) summarizes his own work and the work of other recent research programs in the behavioral psychology of interest, concluding that interest is distinct from curiosity, attention, importance, and intrinsic motivation. He further concludes that no clear 


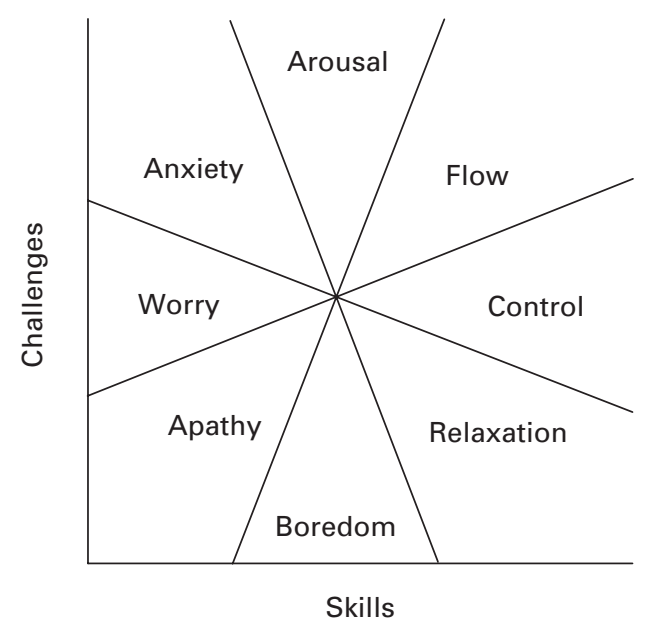

Figure 11.2

Psychological states related to challenge and skill balance (Massimini and Carli).

distinction is warranted between cognitive and emotional interest. What is most relevant to building a complete model of attention is the number of studies he cites demonstrating that "interest promotes the development of knowledge and skills" (209). This should come as no surprise, but it highlights the need to take interest into account as an important variable in the processes that subtend effortless attention, which are so closely associated with skill acquisition. Because current models of attention arise from laboratory studies in which tasks are exogenously motivated, interest rarely plays a part in such studies.

Are there any general elements of activities toward which interest turns attention when engaged in an activity? If activities can be broken down into elements and it is found that certain general elements attract attention, then it may be possible to account for interest and investigate its role in flow activities. But what does it mean for an external stimulus to attract attention? Without an accurate model of attention, this question will be impossible to answer. Therefore, we will turn first to attention and return to specific elements of activities and their relation to interest in the "Implications of the Aperture, Draw, Syntax Model" section below.

\section{Modes of Attention}

One way researchers taxonomize attention is into selective attention and vigilance (Parasuraman 1998b). Selective attention refers to choosing one task of attention from among many, and vigilance to sustaining attention on a chosen task. Selected attention is widely studied by cognitive scientists attempting to map its functional 


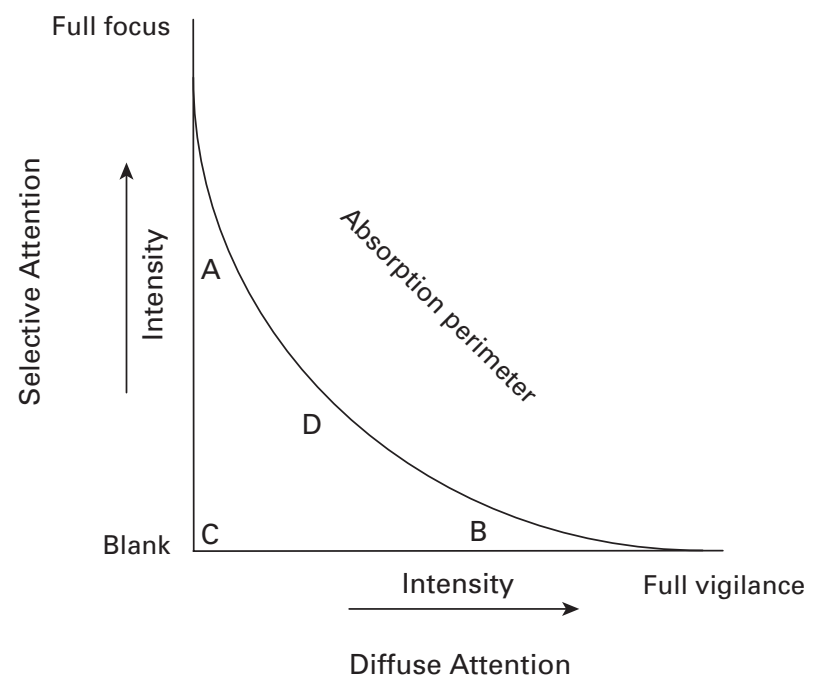

Figure 11.3

Selective versus diffuse attention under normal conditions.

and neurophysiological features, and vigilance is often studied in relation to pathologies, such as attention-deficit/hyperactivity disorder. Here we'll try to understand the relationship between selected attention and vigilance.

When one concentrates on a task, one's concentration may be slight or intense (Kahneman 1973). Let us call intense selected attention focused. The term vigilance presumes a level of success, so let us call the kind of attention that expectantly observes a field of stimuli diffuse attention (Faglioni 1990) and say that high intensity of diffuse attention is vigilance. In a conscious person, these two modes of attention are always simultaneously in operation (Faglioni 1990). Thus, we can run them as axes in a phase-space diagram (see figure 11.3) to analyze their relationship. Diffuse attention begins at blank attention (C), and increased intensity of attention leads to full vigilance (B). Selective attention begins at blank attention (C), and increased intensity leads to full focus (A).

Any position near either axis and toward the blank side seems within the range of normalcy. As one approaches the absorption perimeter, the outer limit of attentional capacity, evidence suggests that attention tends to one axis rather than the other (Parasuraman 1998a), as illustrated-increase in intensity of focus results in a decrease in intensity of vigilance, and vice versa.

A dimension not depicted in this figure is effort. What is the relation of effort to intensity? Intuitively, there would be a direct correlation-the more effort, the more intense one's attention. We could plot the two different kinds of attention against effort as follows (see figure 11.4). 

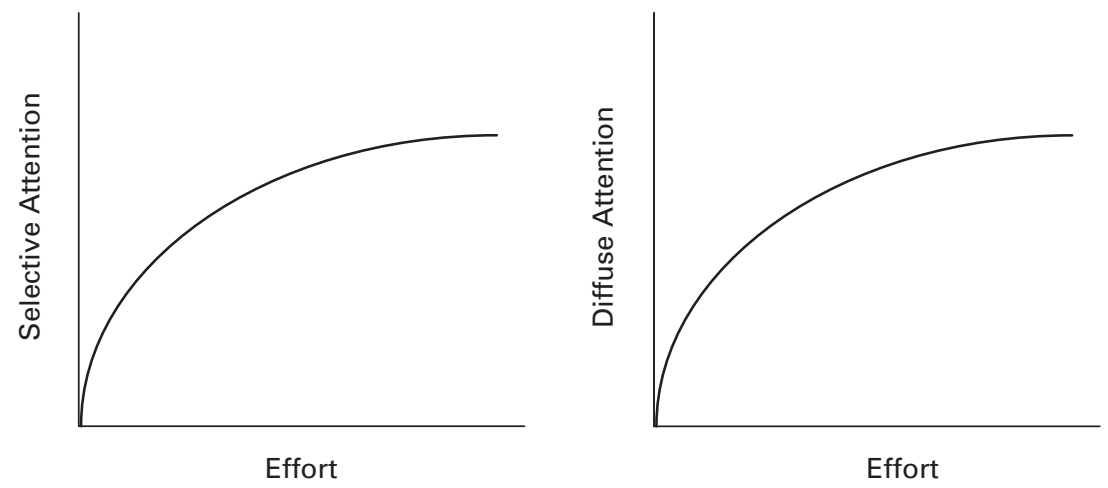

Figure 11.4

Attention versus effort under normal conditions.

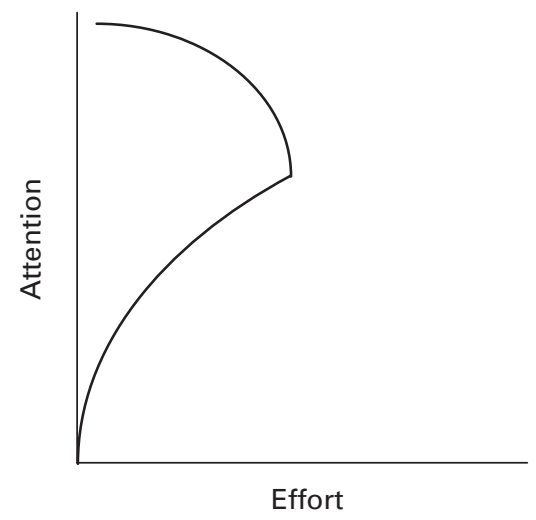

Figure $\mathbf{1 1 . 5}$

Attention versus effort under flow conditions.

In these diagrams, intensity increases with effort until attention asymptotes regardless of increases in effort. An important finding in flow research, however, is that subjects report an increase in absorption and a decrease in effort (Csikszentmihalyi and Nakamura, chapter 8, this volume; Csikszentmihalyi 1975; see figure 11.5).

An increase in intensity over normal circumstances suggests that flow may facilitate the extension outward of the absorption perimeter, and perhaps even the orientation of its parabola (see figure 11.6).

An important dimension not depicted here is the scope of one's attention. While selective attention implies a narrow scope and diffuse attention a broad scope, there is no indication in this depiction of how broad either scope is. For instance, if I am 


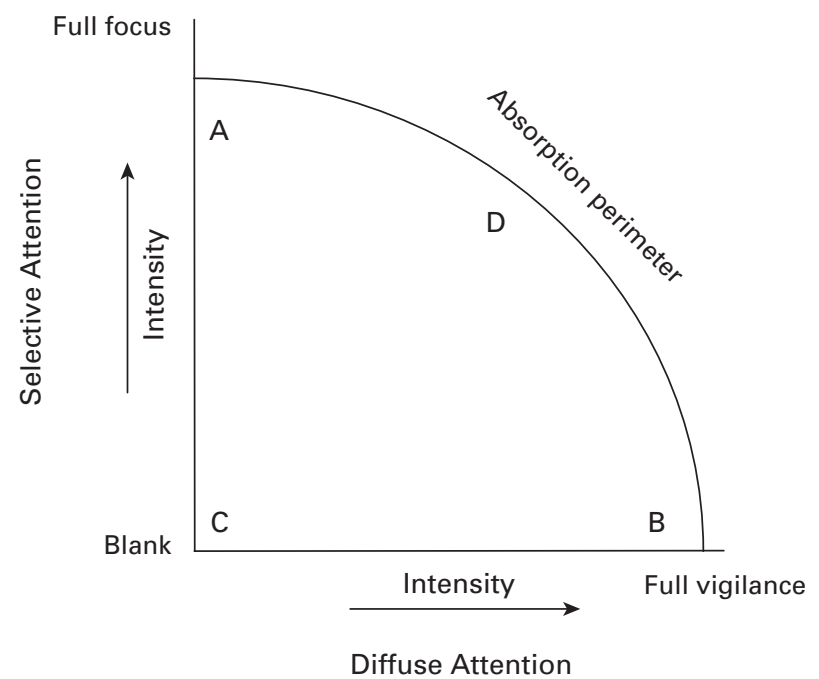

Figure $\mathbf{1 1 . 6}$

Selective versus diffuse attention under flow conditions.

attending to detail $\mathrm{x}$ of activity $\mathrm{M}$ and am vigilant to other potential details of activity $\mathrm{M}$ (suppose my attention is close to point $\mathrm{D}$ ), there is no indication of how vigilant I am to details of activity $\mathrm{N}$. With respect to activity $\mathrm{N}$, it could be that I am closer to point $\mathrm{C}-\mathrm{I}$ am not paying attention to any details of activity $\mathrm{N}$ nor am I vigilant to future potential details. For instance, suppose five men are playing cards in one room while their wives are convening a book club in another room, both groups of spouses within earshot of the other. All could be near point D with respect to their own activity but near point $\mathrm{C}$ with respect to the other.

This observation is consistent with research demonstrating that attention is not an all-or-nothing proposition with regard to domains of activity-that the field of one's attention and the field of one's perception are importantly distinct. Attention is conceived here as domain specific, and all subjects will fall somewhere on the selectivediffuse attention scale with respect to all current and potential domains of activity within their perceptual field. It's not the case that just because a stimulus is within one's field of perception it is also within one's field of attention (Treisman and Gelade 1980).

\section{Apertures and Structures}

What do these modes of attention suggest about the functional mechanisms of attention? Most models of attention focus on the selective aspect of attention, which is 


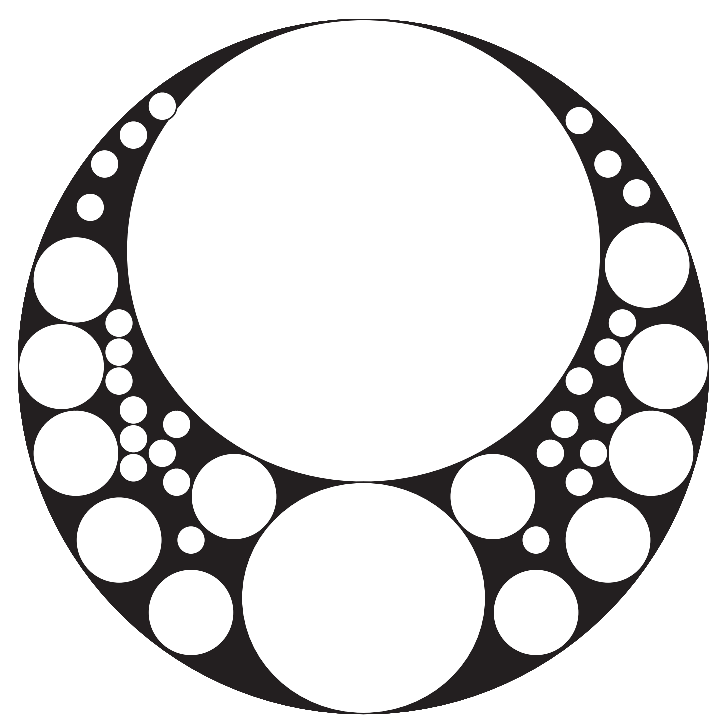

Figure $\mathbf{1 1 . 7}$

Apertures of attention, two-dimensional view.

often depicted as a beacon of light illuminating the object or field of attention. Such a metaphor implies a single control mechanism directing the beam of light and controlling its intensity. Under a typical behaviorist model, the beam would be controlled by external stimuli. Under a voluntarist model, the beam would be controlled by subjective choice. Most such models make room for both "top-down" and "bottomup" mechanisms.

There are other metaphors for selective attention, as well. The common filter theory goes back to Broadbent's Y-shaped tube model (Broadbent 1957). Zenon Pylyshyn (1989) has proposed an INSTantiation FINger (FINST) model of visual attention. These models, and others, generally do not attempt to account for diffuse attention, focusing instead on various features of and controversies around selective attention. I propose that we view diffuse attention as an aperture (see figure 11.7) —not just as a single aperture, however, but as multiple subapertures within one larger aperture-in other words, as multiple avenues of information processing within a single larger avenue. Selective attention will operate within the main aperture only, ${ }^{1}$ although nonattentive processing could be operating in any number of apertures simultaneously.

Attention is understood here as an avenue for the mind to consciously process and assimilate information. ${ }^{2}$ Under the beam-of-light metaphor, the beacon shines on that part of the information that the brain processes in consciousness. Such a metaphor fails to account not only for the separation of information that occurs but also for the 


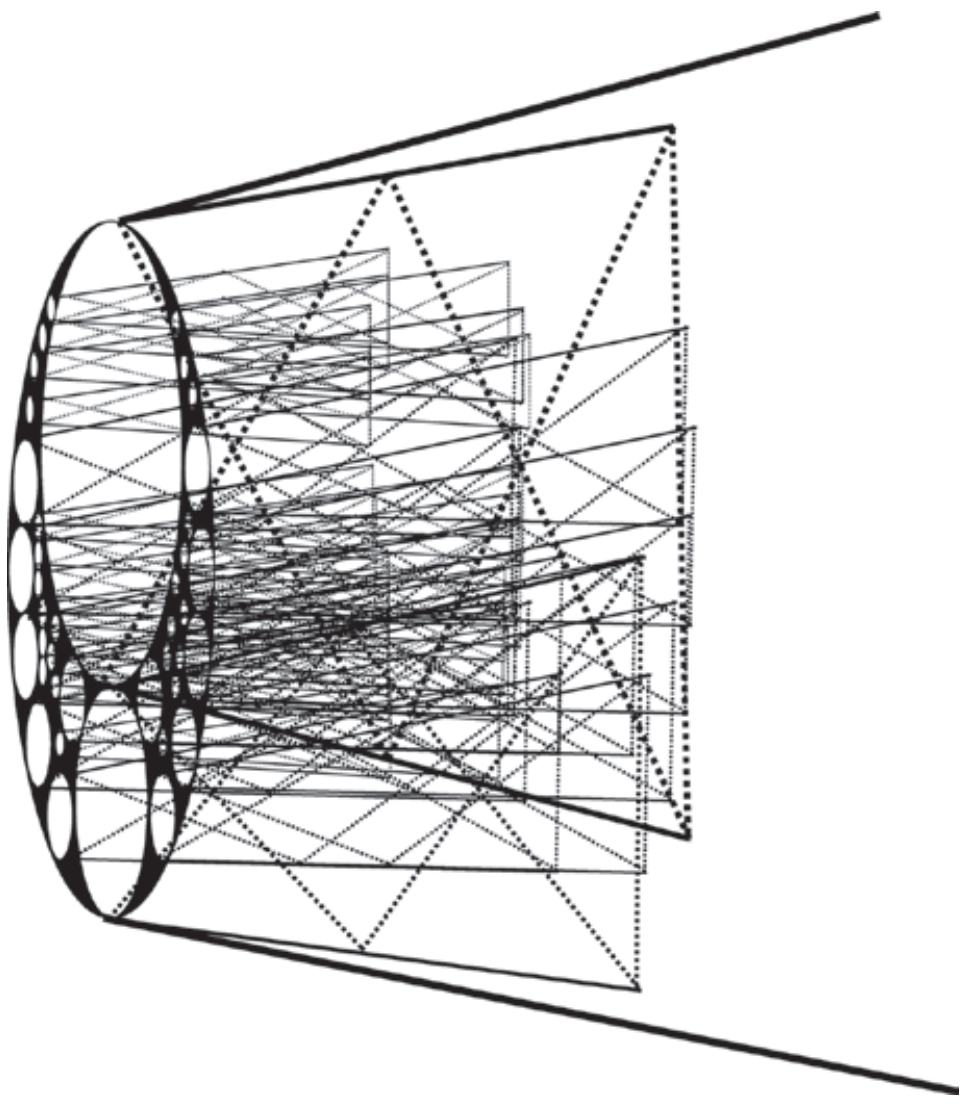

Figure 11.8

Apertures of attention, three-dimensional view.

competing demands of attention, for the negotiation of competing demands, and for special attentive states such as flow and meditative absorption.

Under the aperture model, the outer perimeter depicts full attentional capacity (which may or may not be elastic). Inside this perimeter lie the subapertures of attention. These apertures are not mere windows.

An old, and enduring, idea of the way information is processed is via associative maps, studied in cognitive psychology under such monikers as hierarchical schemata (Houghton and Hartley 1995) and central motive states (Bindra 1974), and recently updated as dynamic connectionist contexts (Botvinick and Plaut 2004). Different terminology has been used over the years. I will call them structures of reference. If we rotate figure 11.7 to peer behind the apertures (see figure 11.8), we will not find a black box with a spotlight shining on a stage but rather a network of structures of 
reference constructed such that each is uniquely sensitized to potential stimuli within overlapping, dynamic domains of activity. The structures are both apertures and avenues of processing.

The glue that binds the elements of each of these structures of reference is their association with each other within a specific domain of activity-one may call it a context. Within a contextual structure of reference (CSR), attenting to an activity involves processing information, setting expectations, evaluating relevant cues, responding through established pathways within the structure, and constructing new pathways according to new developments. The claim is that all conscious attending in human cognition occurs within the context of an activity (see Yuri Dormashev's chapter 13 in this volume for a closely related perspective).

The context is a pathway of processing with links having been established in the past that allow for rapid processing of responses. A context sets expectations regarding what should be attended to (diffuse attention); when a matching cue arises, there is selective attention; relevance of this cue to immediate and long-term goals is calculated according to accumulated information embedded in the structure (long-term memory, habit); a response is formulated and executed accordingly; new expectations arise. This is a rough accounting of what occurs within one CSR aperture pathway. Other CSR aperture pathways will be open simultaneously, and how wide will depend on their relevance to the immediate and long-term goals of the subject. One of the CSR aperture pathways may be termed fight or flight, one may be termed curiosity, and yet another, romance. These are some of the hardwired CSRs, which, no matter how much attention is driven through other CSRs, are likely always open to a degree and ready to expand with a salient cue.

It should be emphasized that the apertures are understood as dilating and contracting in a constant flux as attention shifts from one activity to another over milliseconds. What drives one CSR to gain ascendance over the others, and how is such competition adjudicated? CSRs may be linked to a larger hierarchical goal structure where input through the CSRs goes for further relevance evaluation. Alternatively, they may compete in a Darwinian way for ascendancy, ${ }^{3}$ with the larger goal hierarchy playing some role in which wins out. How factors within the CSRs that drive ascendancy match up with particular internal or external stimuli (Botvinick and Plaut 2004) must also be an integral part of the process. My use of the term structure may lend an unintended sense of stasis to the model, but these structures are envisioned as dynamic -in constant flux with constantly shifting boundaries. Thus, an element within one structure may also belong to other structures.

Let us look at an example. A young man in a technologically primitive society (say, in the highlands of Borneo) is on a lookout platform in the forest. ${ }^{4}$ His job is to lash some spearheads to spears while keeping watch for any sign of marauding warriors from another tribe. He is new to both tasks and not expecting much excitement. When 
taking up his position, he surveys the surroundings. At this point, a CSR aperture pathway that we might call defense opens widest. Expectations are raised for signs of danger that he has been taught or has encountered before, all of which have been integrated into this pathway-such things as birds taking flight, sounds of snapping twigs, signature hoots of neighboring tribes, and so forth. This CSR has primed him to respond with urgency to such cues.

After surveying the surroundings, he squats down to begin lashing a spearhead to a shaft, a task he has only seen others do and is now trying for the first time. His defense CSR contracts, and his "spear-making" CSR dilates, but this CSR is still rather empty, and this lack of skill makes it difficult for him to recognize and adjust to appropriate cues in the task. On top of this, the previous evening he had had a riveting encounter with a young woman at a ceremonial dance. His romance CSR keeps crowding out his spear-making and defense CSRs.

While making his first spear and daydreaming about his love interest, the defense CSR, though comparatively small, is still open, and before the first spear is complete, the sound of a snapping twig catches his attention. The romance CSR immediately contracts, crowded out by the dilating defense CSR. Without moving, the boy scans the surroundings with ears and eyes. A flock of birds takes to the air. He identifies this through his defense CSR as another danger signal. He hears a hoot that he can't place-another bad sign identified through the defense CSR. These accumulated dangers prompt his defense CSR to temporarily integrate with his fight-or-flight CSR, and his heart begins beating faster, his adrenaline flows, and he realizes his unfinished spear could be very useful if finished. The integrated defense/fight-or-flight CSR is near full dilation until he turns attention to the nascent spear, at which time his spearmaking CSR dilates, forcing contraction of the integrated defense/fight-or-flight CSR. ${ }^{5}$ Both CSRs contract and dilate as he shifts attention back and forth. The more he concentrates on his spear, the less he is able to attend completely to signals from the forest, and vice versa.

We see in this example that selective attention is activated within an activity domain which drives diffuse attention. The temporary persistence of diffuse attention allows for the integration and assimilation of relevant information via selective attention.

A more contemporary example would be a male college student at a football game. Actively competing CSRs would likely be socializing, analyzing the game, cheering, and sustenance. ${ }^{6}$ Other CSRs that would simultaneously be open but minimized might be unfinished homework and, of course, romance. Imagine if, to the student's surprise, a professor or a friend of his (stern) father sat down next to him. Now, in addition to his socialize with elders CSR's dilating, it will elide with his socialize with friends CSR, channeling his responses away from remarks and behavior suspected to be deemed distasteful by his neighbor. 
To be more specific, imagine two alternative versions of this scenario. In one, before the elder arrives, a referee makes a controversial call unfavorable to the home team. The male fan in question, gesticulating threateningly toward the field, shouts a string of obscenities. In the alternative version, the same call is made but after the elder arrives, in which case the student's response would be more mild: "I can't believe that," throwing his hands up. Same cue, different response, guided by a qualitatively different CSR.

Now suppose something salient occurs, such as a siren blaring. Salience is often considered the mark of an entirely externally driven stimulus. The current model, however, would require that every attended cue be processed through a CSR and be dependent on two things: the availability of the CSR with respect to other CSRs and the sensitivity of the stimulus within the CSR. Depending on the listener, a siren could conceivably enter through a curiosity CSR (as with a young child), through a public safety CSR (for a distant motorist), or through a danger CSR (for a criminal). At a football game, it may enter through a cheering CSR. The CSR through which it enters depends on one's prior experience. For someone new to football games, it would likely enter through a danger CSR and then be integrated into a cheering CSR relevant to the football game. The actual intention of the siren operator would bear no necessary relation to the CSR aperture pathways of listeners, but the most seasoned fans would be the quickest to channel the sound through the football CSR and respond appropriately. A particularly nerdy, though seasoned, fan sitting in the upper bleachers concentrating on calculus problems might have the siren enter through a cheering CSR and not attend to it at all—not even remember hearing it if questioned about it later.

Again, salience, under this model, is not an independent variable in attention and is, instead, predicated upon CSRs. It may be true that we are hardwired with certain CSRs, such as fight or flight, through which loud noises are attributed salience. But it is also true that such noises will be selected for attention depending only on their relevance within a CSR and the status of other competing CSRs. A straightforward way of challenging the notion of the inevitability of salient stimuli is to consider external context: A neon Coca-Cola sign will be much more salient in the middle of farmland than in downtown Tokyo. Another way to challenge it, however, is by considering internal context: The bouncing cheerleaders on the sidelines of the football game will be more salient to our heterosexual male fan than to his homosexual friend, who through his romance CSR finds more salience in the physiques of the players running and jumping on the field.

\section{Draw}

The postulate above is that the internal conditions of CSRs, in conjunction with relevant external stimuli, drive attention. As such, the main drivers of attention are 
internal rather than external. A salient phenomenon in the environment is best characterized not as a stimulus (thereby implying a behaviorist response) but as a cue, implying that there is a preexisting structure of responsiveness sensitized to the recognition and processing of the phenomenon.

One could try to argue counterfactually that without the stimulus there would be no attention to it and therefore attention begins in the stimulus, but if it is true that the CSR is always open to some extent and that it is appropriately sensitized to external phenomena, then stimulus or not, there is always diffuse attention. Ruth Garrett Millikan, discussing language and cognition under a theory she calls biosemantics, suggests that we do best to understand biological organisms not as representation producers but as representation consumers-a representation has meaning only as understood by the system-so consumption is primary (Millikan 1989). Likewise, I would argue, a stimulus has the potential to be attended to only insofar as it can be assimilated into and processed by an open CSR.

If the salience of external phenomena is dependent on internal constructions, how are we to conceive of the flow of information? Traditionally, sense data are conceived to impinge on our senses like so many projectiles flying through space; our sensory organs let them in, and then our processing pathways filter some out and let others through. The problem with the projectile view of information flow is that the information is, again, originating externally. What many studies in inattentional blindness (Mack, Pappas, Silverman, and Gay 2002; Mack and Rock 1998) have shown, however, is that often we simply do not see phenomena that are obviously open to view. Mack and Rock $(1998,228)$ conclude that the most important factor in an object's capturing attention "is the meaningfulness or signal value of the stimulus." In other words, it is not that irrelevant stimuli are filtered out but that only relevant stimuli are drawn in.

The projectile view of information flow presupposes that the information will be processed unless rejected. The view presented here is that there is no information until there is a CSR ready to assimilate and process it (e.g., if NPR is being broadcast in the forest and there is no one around to hear it, there is no news there in the forest). Does this open me up to a chicken-and-egg objection? No, because an evolutionarydevelopmental view of the human being posits a certain amount of hardwiring that begins with rudimentary CSRs and allows for them to gradually mature and differentiate (Gopnik and Schulz 2004; Millikan 1989).

I introduce the notion of draw as a way of emphasizing the fundamental role of sensitivity in attention. There are two basic claims: (1) We attend only to those phenomena to which we are cognitively capable of attending, and (2) once we are capable of attending, there is an active internal mechanism that struggles for predominance against other active internal mechanisms to receive and process relevant information. 
How can we make sense of a cognitive draw? How can there be a pulling force from inside the head that acts on things outside the head? Action at a distance was a common problem in early theories of physics-how could it be, for instance, that electricity suddenly flows through a channel when there is nothing obviously pushing it along? The answer lies in there being a particular difference between one place and another. If the voltage on one end of an electrical channel is identical to the voltage on the other end, there will be no flow of electricity. Only when the voltage increases on one end do electrons flow toward that end. One way of understanding it is that the voltage differential establishes a draw that enables the flow of electricity. Just as voltage is known more accurately as electric potential, attentional sensitivity in cognition could be conceived as information potential.

Electricity isn't the only occurrence of a draw due to a differential in a potential channel of a flowing medium. A similar set of phenomena power the flow of air in the form of wind. Aside from global processes, such as the Coriolis force, wind is generally understood as the flow of air through a channel of pressure differentials, from a high-pressure area to a low-pressure area. One way to understand it is that the low-pressure area sets up a draw from the high-pressure area. For instance, when a window breaks in movie depictions of airplane disasters, the reason that the air is depicted as being sucked outwards from within the plane is that the air pressure at an altitude of 33,000 feet is much lower than the artificially pressurized cabin.

A third way to think of draw is through economic demand. The analogy of cognitive information flow to economics is felicitous in that the appearance of a product on the market originates from the supply end, just as stimuli originate externally to the agent. However, just as a product will not move through market channels until there is the draw of consumer demand, so information will not flow through cognitive channels until there is the draw of attentional sensitivity.

We see still more examples of the action-at-a-distance effect of draw in the cases of heat and dissolved substances. It may be best to think of raw sense data (sound waves, photons, etc.) as the channel of the information stream, the infrastructure, that is always there and of information as the medium that flows through the channel. Attention is not a sensitivity to photons but to the information that photons carry.

The metaphors, whether explicit or implicit, of attention as a spotlight and of sense data as projectiles that would strike and register if not filtered are pervasive in the cognitive science literature. What differentiates the model expounded here is the realization that attention follows not sense data but rather information. Broadbent (1958) distinguished between stimuli and information some five decades ago, but the idea still persists today that we will be overwhelmed (even by information) without a filtering mechanism. Briefly going back to the beginnings can help put this idea to rest. 
A simple stone is bombarded by sound, heat, and the entire range of stimuli from the electromagnetic spectrum. Does it feel overwhelmed? Of course not. When life formed and successfully opened the first window of sensitivity to its environment, was it overwhelmed with so much incoming sensory stimuli? No, because the window was open only to a single kind of information that was ultimately adaptive. To everything else, it was like a stone. As creatures became more complex, new sensitivities opened up to enable creatures to process new information, but only to the extent that it was ultimately adaptive. At no step was there a gush of data that required filtering, and that goes for humans, too. William James gave us the term "blooming, buzzing confusion," and we have the common intuition that when babies are born they are overwhelmed by sensory stimuli that they cannot interpret. It's more likely, however, that infants are born with a limited number of active domains of activity within which they are naturally responsive to what we would consider primitive information. Sensitivity and responsiveness would then expand in tandem.

As our sensitivity expands within domains of activity, the specific circumstances combined with specific internal conditions of the CSR together select information as needed. There is no perceived bombardment and no filter. Pylyshyn's (1989) FINSTs that point outward, index, and track features of visual stimuli are similar in that they are endogenously prepared to respond to specific kinds of features of the external world rather than accepting everything and then dumping what is not needed.

\section{Syntax}

The claim is made above that all human attention arises within a domain of activity, so it is important to inquire into what is meant by the term activity. An activity is understood here as a set of constraints that are related in such a way that they facilitate the accomplishment of a goal. ${ }^{7}$ This set of related constraints is understood as a syntax because it provides a dynamic structure for an unfolding concatenation of actions (including thoughts). ${ }^{8}$ The syntax of daily etiquette is implicit, whereas the syntax of chess is more explicit, with its large body of rules, but the suggestion here is that the syntax of any activity goes beyond any explicit set of rules to encompass all constraints on thought and action relevant to the activity as a situational, temporal, autopoietic enterprise. Thus, the syntax of competitive downhill skiing includes not only the international rule book but also the resistance of its moguls and gates and the implicit rules of socializing among competitors.

Constraints are parameters within which one may respond to a cue. ${ }^{9}$ Cues are recognized according to the total set of constraints, the syntax. How good one is at negotiating a syntax will often depend on one's amount of experience within that syntax. Cues gain and lose salience accordingly, and responses become more or less automatic accordingly. Cues are phenomena the appropriate response to which 
advances one toward a goal. They can be external or internal, perceived or cognized, and sensitive or insensitive to response. A cue can be a rock on a cliff face, a move in a mental chess game, a batting eyelash, and so on. Each of these cues is recognized within a syntax, and a response follows according to the constraints of that syntax.

The final element in this formulation of an activity is the response. Once the goals and syntax are organized in a functional way and one is able to recognize relevant cues, two things occur in cognition: predictions are made, according to which responses are primed to possible future cues, and immediate responses are executed (the effects of which may become future cues). This should not suggest, however, that potential responses are temporally secondary to the recognition and assimilation of cues. The capacity to respond - the syntax of response-may play an active, and even necessary, role in establishing the initial sensitivity to the cue, itself (see Borghi 2005; Hommel, chapter 5, this volume). A response would then be functionally inseparable from a cue, even though in real time a response may be temporally separate or may not even arise at all. In this sense, a cue would entail at least a potential and approximate response in order to be a genuine cue. This conceptual necessity linking response to cue, does not, however, mean that the response is actually necessary, or that a nonhabituated action may not be identified post hoc as an appropriate response.

For everyone but a newborn (perhaps even a newborn), every activity involves some amount of prior cognitive habituation. Habituation is the Hebbian facilitation of response through repetition to a general cue within the general constraints of a syntax and according to generalized situational demands. An initial step in the habituation process is recognizing a cue as general-as something the likes of which may occur again under similar circumstances and require a similar response. A related step, formulation of a response, is established in the same context. If the context is novel, response formulation will happen haphazardly at first, arrived at by navigation through related but not identical CSRs, which elide in and out until appropriate responses are arrived at and incorporated into a nascent new CSR. Habituation is viewed here as the construction and reinforcement of the pathways of a CSR.

In addition to syntax, goals, and response, the final element of an activity relevant to this model is how the response is executed. William Calvin posited the notion of the ballistic thought (Calvin 1993), suggesting that cognition involved in time-constrained, complex serial actions is a borrowing of the same cerebral mechanisms that allow us to accurately hurl a projectile at a target. Launching mental calculations is like launching a series of actions that once started cannot be adjusted via feedback and response, because the response time is too long. I suggest that the real-time negotiation of the syntax of a CSR is just this kind of ballistic thought, except that in an activity, it is more open to revision than in an action, itself. It's difficult to think of a human response that is not in some sense ballistic, that does not involve the initiation of a concatenation of cerebral activity that proceeds in some way on its own. 
Habituation involves the automation of the links in the concatenation. Thus, each of the three steps of response involves the possibility of habituation: the recognition of cues (what to be sensitive to), choosing a response (why this response and not another), and the cognitive and motor activity that are the response (how to execute it and when-i.e., timing). The provisional answers to the what, why, how, and when of an action are the syntax of a CSR and allow for the smooth unfolding of the perception-action cycle.

\section{Implications of the Aperture, Draw, Syntax Model}

As I suggested at the start of this chapter, adopting any particular model of attention will entail specific available inferences from the model. What is the payoff for using the ADS model? I think there are many.

Given the elements of an activity-the goals, the syntax, and the response-we can begin to envision a theory of optimization of action. The obvious avenues of intervention are at any of the four opportunities in the process of habituation of a response just noted: the what, the why, the how, and the when. These may be more or less important depending on the weightings of certain aspects within a syntax. In chess, the why is most important; in figure skating, it is the how; in urban warfare, it is the what; and the when is crucial in all of these examples (though less obviously so in chess).

As discussed, the use of the term syntax should not give the sense of an intransigence to modification. Not only is a CSR constantly open to modification but its place in the competitive hierarchy for attentional predominance is also constantly open to modification. As a CSR becomes richer through experience, assuming at least a moderate level of associated positive affect, it will compete more successfully for attention, and an interest may develop.

In this model, interest is best defined as motivation through enjoyment. An underappreciated corollary to Csikszentmihalyi's theory of flow is his distinction between enjoyment and pleasure. The full passage is worth rehearsing:

Playing a close game of tennis that stretches one's ability is enjoyable, as is reading a book that reveals things in a new light, as is having a conversation that leads us to express ideas we didn't know we had. Closing a contested business deal, or any piece of work well done, is enjoyable. None of these experiences may be particularly pleasurable at the time they are taking place, but afterward we think back on them and say, "That really was fun" and wish they would happen again. After an enjoyable event, we know that we have changed, that our self has grown: in some respect, we have become more complex as a result of it.

Experiences that give pleasure can also give enjoyment, but the two sensations are quite different. For instance, everybody takes pleasure in eating. To enjoy food, however, is more difficult. A gourmet enjoys eating, as does anyone who pays enough attention to a meal so as to discrimi- 
nate the various sensations provided by it. As this example suggests, we can experience pleasure without any investment of psychic energy, whereas enjoyment happens only as a result of unusual investments of attention. (Csikszentmihalyi and Csikszentmihalyi 1988, 46)

A person is interested in activities that lead to enjoyment but not to pleasure. One is interested in gardening but not shooting heroin. Drinking wine in an enjoyable way may further develop an interest that enriches the enjoyment. Drinking wine in a pleasurable way leads to inebriation. Enjoyment and pleasure are not mutually exclusive, but interest is associated with enjoyment, not pleasure. Further, people are not interested in activities that do not yield any enjoyment whatsoever. Researchers who study interest confirm that interest is closely associated with the understanding of enjoyment offered here, but because they do not make the enjoyment-pleasure distinction, conclusions in the literature state that "interest and enjoyment are distinct positive emotions" (Silvia 2006, 29). The confluence of enjoyment and interest, in my view, is that they both enrich CSRs in a positive way.

There is obviously a tight correlation, probably a mutually reinforcing relationship, between interest, enjoyment, and flow. Achievement of one, therefore, likely yields achievement of the others, suggesting that if one wishes to cultivate or increase interest in an activity, engendering flow would be of some assistance. Achieving flow intentionally, however, can be elusive. For this reason, it would help to understand potential obstacles to flow.

Because flow depends on a balance of challenge and skill (and assuming timely feedback), any imbalance would diminish chances of achievement. Thus, two primary obstacles to flow are high challenge/low skill and, conversely, low challenge/high skill. A third obstacle is distractions (internal or external), and finally, even if there is a balance of challenge and skill and an absence of distractions, flow may still be difficult to achieve if there is a lack of interest. There we are back at interest.

In most activities, when the first two obstacles are encountered, adjustments in challenge level are the easiest to make, and practice results in an increase in skill. The most difficult adjustment to make is when the level of skill is too high in relation to the level of challenge, as in monotonous assembly-line or office work. Csikszentmihalyi relates the story of an assembly-line worker who increased the challenge level of his work activity by fabricating personal challenges with relation to the demands of his job (Csikszentmihalyi 1990, 39). Something similar can be observed in children at play, and this may actually be one of the functions of play-to build CSRs through incrementally more difficult challenges.

Why does Johnny climb on the low walls, avoid cracks, and kick rocks while walking home from school, instead of walking like a typical adult? One explanation is that he increases the level of challenge. He has already mastered walking, and just walking is boring - there is no sense of enjoyment. By fabricating challenges and meeting those challenges, he increases his level of enjoyment, in addition to his skills 
of balance, locomotion, and so on. This natural desire to meet challenges and the natural satisfaction gained from achieving goals may go a long way in explaining why children learn so readily. It also goes a long way in explaining how to overcome the obstacle of low challenge.

At the level of the CSR, the introduction of new challenges adds complexity to the activity's syntax. A sports coach, for instance, keeps the attention of players while emphasizing fundamentals by creating a number of high-challenge training exercises and keeping the players moving from one exercise to the next. However, this process is extrinsic to the players. To make the activity autotelic, the athlete must, himself or herself, incorporate such challenges into his or her own CSR. The self-motivated athlete must work on the cognitive level as well as the somatic-building challenges into an otherwise boring workout routine.

Distractions are an interesting case and a good illustration of the usefulness of the aperture model of attention. Under the aperture model, when one is fully engaged in an activity, that activity's CSR aperture pathway widens until nearly all attentive processing capacity is monopolized by that CSR, blocking out competing CSRs-the would-be distractions. Competing CSRs can distract only when the desired activity's CSR has not grown to ascendancy or when that ascendancy cannot easily be maintained.

William James said that there is no such thing as sustained attention, only attention renewed. This is consistent with the aperture model of attention in which different CSRs are competing for ascendancy under normal conditions and, from a subjective point of view, it therefore takes effort to sustain (repeatedly renew) any one CSR for an extended period of time. I suggest that when a CSR is opened to ascendancy, its predominance is usually precarious because it is vulnerable to other CSRs' attempting to simultaneously dilate. Mental effort can be understood as the deployment of cognitive resources to renew conscious attention to an activity. The more vulnerable an ascendant CSR is relative to the strength of competing CSRs, the greater the effort required to maintain attention. One can analogize the process to pedaling a bicycle. To maintain momentum on a flat surface (normal conditions), one must exert energy by pedaling. To maintain momentum on an upward incline (distracted conditions), one must pedal harder. To maintain momentum going downhill, however, no expenditure is required. Flow activities, by monopolizing attentive capacities, achieve a stable momentum such that they endure without external propulsion and so can persist despite incursions from other CSRs. Effortless attention, then, is constituted by the autotelic maintenance of a CSR. The syntax is so tightly constructed, the facility of responses so automatic, and the information flow so large, that the momentum created dominates the entire perception-action cycle, thereby inhibiting the rise of other CSRs.

As discussed above, the fourth obstacle to flow-lack of interest-is related to enrichment of a CSR and positive affect from enjoyment. Interest in activities is often 
neglected as a subject of study, I believe, because it is understood as subjective, like taste: To say that one is interested in baseball is like saying one has a taste for spicy food or impressionist painting. As such, it is written off, like taste, to unanalyzable personal preference (see Dormashev, chapter 13, this volume for a different and more subtle view of personal taste, which is closer to my notion of interest). Interest, I am suggesting, however, increases in an activity with the complexity of one's CSR (assuming concomitant positive affect). But what are the ways in which one's CSR may be complex and increase in complexity? There may be many; below I describe two: formality of actions and syntax of actions.

Formality in an action refers to the narrowness of the parameters of movementthe lower the amount of flexibility (the fewer correct ${ }^{10}$ ways to make the movement), the higher the formality. Consider dance. For teenagers at a high school dance, the degree of formality in any one movement is low. For a traditional ballerina, however, the degree of formality in each movement is extremely high-there are precise ways in which to execute each movement, and going outside those parameters would be perceived as incorrect.

Syntax in a set of actions refers to the parameters constraining how one action is concatenated with the next and is directly related to syntax in a CSR; since CSRs are functionally intermodal and internal cognition eventuates in external action, it makes sense that syntax in an action is part of the syntax of a CSR. Again, consider dance. For teenagers at a high school dance, how one strings one action of the dance to the next generally makes little difference, and so the degree of syntax is low. For the ballerina in a tightly choreographed classical dance, however, there is a very high degree of syntax, and if one move is out of order, even if the formality of the movement is executed with virtuosic brilliance, it will be perceived as incorrect.

Let us distinguish an activity from an action. Swinging a bat is an action, while playing baseball is an activity. We may categorize all activities based on the degree of formality of the actions that constitute them. Imagine a continuum with completely formal activities (i.e. those composed of highly formal actions), such as choreographed dance, martial arts forms, or acrobatics, on one end. On the other end will be completely informal, or open-ended, activities (those with informal actions), such as a high school dance or eating a grape. ${ }^{11}$ Most activities will have both formal and informal components, and, therefore, fall somewhere between the two extremes. Entertaining guests, for instance, would appear toward the informal end, while driving and making pottery might appear somewhere in the middle.

We may also categorize activities according to another continuum, namely, the amount of syntax involved in connecting the actions together within the activity. Activities with a higher degree of syntax may be called more syntactic, and those with a lower degree of syntax less syntactic. We can also place activities on this continuum with respect to others. For instance, a classical ballet dance would be highly syntactic, 


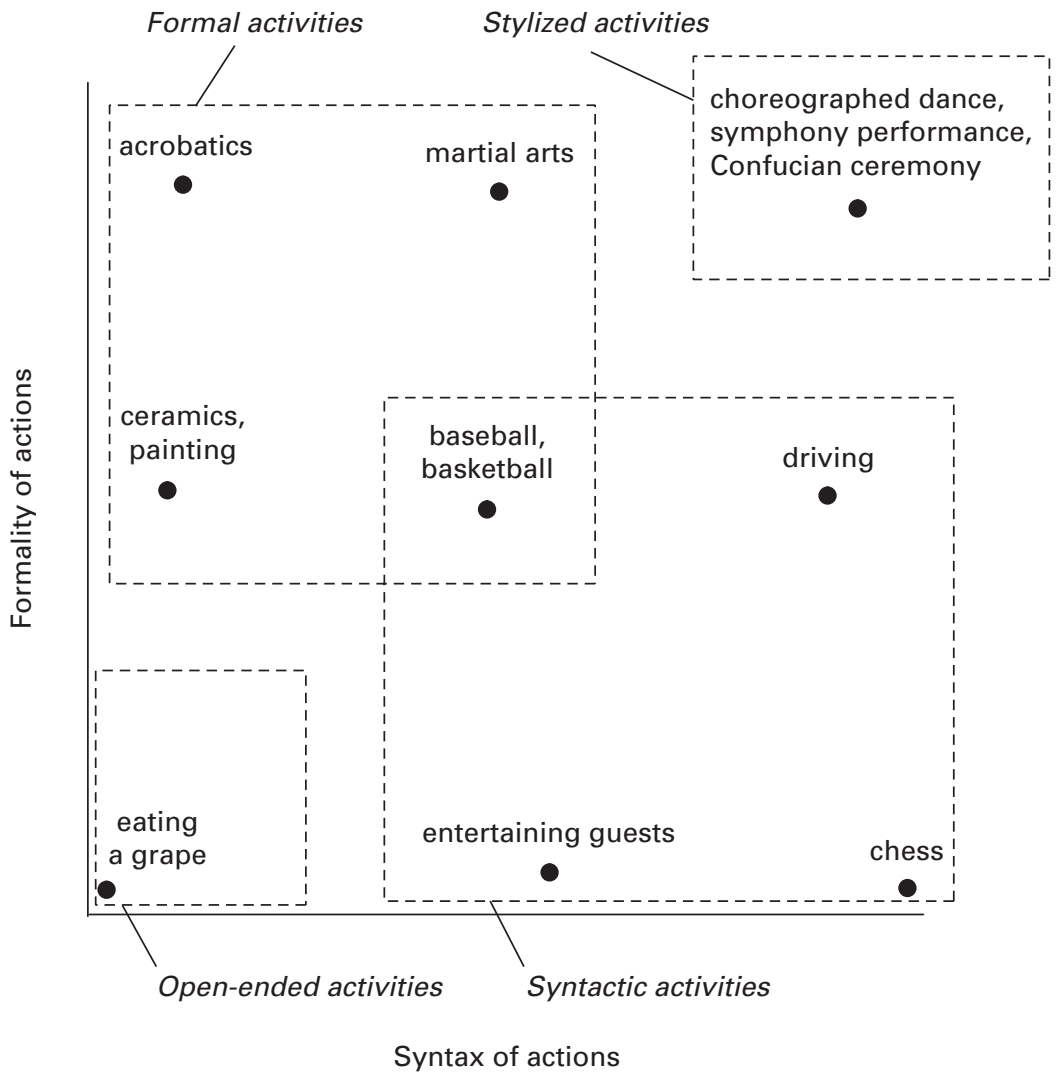

Figure 11.9

Action diagram: formal versus syntactic activities.

as would chess and, perhaps, driving. Entertaining guests and executing martial arts moves would be moderately syntactic, and eating a grape, making pottery, and executing unchoreographed acrobatic moves less syntactic.

Let us now combine these two continua into two axes in a phase-space diagram (see figure 11.9)—formality being the vertical axis and syntax being the horizontal axis. Fully formal and highly syntactic activities, such as a symphony performance or a highly ritualized ceremony, will fall at the top right, while informal nonsyntactic activities, such as eating a grape, will fall at the bottom left. Elsewhere on the grid, we will find driving (middle right-lots of syntax, less formality), martial arts practice (top middle-lots of formality, less syntax), chess and other games (bottom right-lots of syntax, very little formality), painting, sculpture, and other arts (middle left-some formal techniques but little syntax), and baseball and other sports (center-a moderate 
amount of both formal actions and syntax). We can now identify activities as belonging to one of four groups: formal (top left), syntactic (bottom right), stylized-both syntactic and formal (top right)—and open-ended — neither syntactic nor formal (bottom left).

Studying this diagram, one may surmise that among the general populace flow is most commonly found in syntactic and formal activities. Activities with the highest probability of engendering flow likely fall at the intersection of these two squares, where we find such activities as playing popular music, ballroom dancing, and all kinds of sports. Outside of these two squares, in the stylized activities and the openended activities, is where we find flow the most difficult to achieve, on the one hand because challenges are too high, and on the other because challenges are too low.

With the help of this way of conceptualizing the relationship between activities and flow, we can get a bit more purchase on the question of overcoming obstacles to flow. Consider, first, what it takes to achieve flow in open-ended activities, such as leisurely walking to a destination, washing the dishes, taking a shower. In activities such as these, in which the degree of formality and syntax are so low as to present very little challenge, other CSRs easily usurp predominance because there is not enough challenge in the formality or syntax of the actions to maintain momentum. In typical flow activities, habit is an ally because it allows you to execute part of a complex action or set of actions while paying attention to other, more subtle, features of the activity. In open-ended activities, habit is the enemy because there are no subtleties of form or syntax of the activity to move on to.

We discussed above that introducing artificial challenges to an activity is one way to overcome low challenge. Another way is through the practice of mindfulness. Mindfulness is a form of meditation in which one keeps various elements of an otherwise habituated activity in attention. Meditation, in all its stripes, does one thing well-it destroys habits of mind. One of the most intransigent and difficult to identify habits is the very process in which attention transitions rapidly, successively, repeatedly, and unnoticeably from one CSR to the next (let's call it attention substitution). Traditionally, the first step in Buddhist meditation is to recognize the process of attention substitution (phrased in different terms) and prevent the attention from following the ascending CSR. Attention substitution is easy enough to experience. Simply sit still and do nothing, paying attention only to your natural breathing and noticing how often your attention shifts to something else. Each "something else" is a competing CSR.

In this breath meditation, one has formed a meditation CSR, which is so devoid of both formality and syntax that it is nearly empty of any content, whatsoever. Of course, this is difficult to maintain against other more established, richer, or more urgent CSRs. The method of this kind of meditation more generally (terminology varies, so I'll call it open meditation for simplicity) is to allow a competing CSR to arise 
but then to abandon it and come back to the meditation CSR. In effect, this type of meditation CSR is a meta-activity that takes attention, itself, as its subject. There are two important ways in which the activation of CSRs is habituated. One lies in how various CSRs are interlinked, and so repeatedly moving from one to the next by virtue of these connections will be habituated. The second way is through the very process of allowing different CSRs to arise one after another, which is a deep habit that goes back to infancy. The practice of abandoning a newly ascendant CSR serves to dishabituate the meta-activity (attentional substitution) in which CSRs spontaneously compete with each other, to diminish the chance of the ascendant CSR's attempting to gain ascendance again-like an action that fails to receive positive feedback. Over time-weeks, months, and even years-the meditation CSR, during the activity of meditation, expands to monopolize all of attention, and competing CSRs fail to arise. The anomaly of this kind of meditation is that it can be maintained despite so little apparent complexity of content and so little information flow; however, this is, of course, explained by the understanding that the activity of attentional substitution has itself been modified such that competing CSRs no longer compete as vigorously.

From open meditation, let us return to mindfulness meditation, a form of meditation adopted during active life. Mindfulness is said to be most effective when it is coupled with sitting meditation as just described. If competing CSRs have been muted through the successful cultivation of a meditation CSR, then when performing normal habituated tasks, competing CSRs are less likely to arise. As mentioned above, actions naturally concatenate in syntactic series, and in the process of habituation these cognitive processes are tucked away for automatic processing. Mindfulness practices bring these processes back to the foreground. While walking, one notices one's balance, one's gait, one's breathing, and as many elements of these that one can bring to mind. The challenge lies in identifying these elements, attending to them in turn, noticing subtle characteristics, and even fine-tuning them. In this way, one reconstructs the CSR, enriching both its syntax and formality, moving it from an open-ended activity further toward the center of the action diagram, where enjoyment and sustained attention will more likely ensue. This is how mindfulness mediation can assist in achieving flow in low-challenge/high-skill situations.

In this section, we have circled back to the topic of the first section ("Interest and Effortless Attention")- the neglected role of interest in accounting for effortless attention in flow. The original intention was to begin to see how the ADS model of attention can yield new insights into the diverse phenomena associated with attention. This was done by further explicating interest with respect to CSRs and then considering how interest, enjoyment, and flow may reinforce each other in activities that have a moderate amount of formality and syntax of actions. Also, not only did the model make sense of how mindfulness exercises can be useful in raising the level of challenge in order to facilitate flow but it also made sense of meditation, itself. Finally, this 
model helped account for the phenomenon of effortless attention itself, conceived as the highly automated negotiation of a tightly constructed, complex syntax with a very high flow of information. Combined, these provide a momentum in a CSR that inhibits competing CSRs.

\section{Conclusion}

The purpose of presenting the formality-syntax diagram (figure 11.9) is to demonstrate how understanding attention via the aperture, draw, syntax model can lead to a more fruitful theoretical engagement of features of cognition that are associated with attention. As discussed, effortless attention becomes quite a bit less mysterious when it is understood as the inhibition of competing CSRs in the normal process of attention substitution, either by momentum of the ascendant CSR, as in flow, or by the diminution of the substitution process, itself, through meditation. The hope is that this model will open up new avenues of profitable exploration for future research on attention.

\section{Limitations of This Model}

One obvious limitation of this model is that it claims no direct correspondence to neurophysiology, and for this reason, it may be open to objections from readers attempting to apply it to human neurophysiology. For instance, one might object that neurophysiologists have demonstrated that attention is not a unitary phenomenon, and yet the ADS model of attention presumes that it is. I believe these confusions can be cleared up because although this model is merely a functional model, the aim is to account for human cognitive functions that are underpinned by neurophysiological processes. Thus, any neurophysiological processes that underpin functions that contradict it would invalidate it. In the case of the nonunitary nature of attention, the ADS model posits not that there must necessarily be one physical location in the brain that is a channel of all attentional processes but that the neurophysiological processes that underpin attention act as one functional channel, as if there were only one physiological channel. Just as physicists include a notion of a center of gravity in models of physical motion even though one cannot locate a center of gravity in the physical universe, so this model posits a central channel of information flow even though no such physical channel exists in the brain.

Another limitation is that this model is purported to apply to conscious attention, and consciousness is a notion only tenuously supported by the cognitive scientific literature. By "conscious," I am simply referring to the explicit-implicit, controlledautomatic distinction common in the literature (but see Blais, chapter 6, this volume). I am suggesting that in this model of attention, there is neither explicit nor implicit activity alone but necessarily both together. If it were all implicit, there would be no attention, as such. (This is not to say that there would be no processing. It is a matter 
of stipulation-attention to one's actions is understood here as involving awareness of some of the relevant events, by definition.) If it were all explicit, the perceptionaction cycle would slow to a snail's pace.

Finally, the model is limited by the lack of empirical data supporting it, particularly with respect to the formal-syntactic distinction in the last section. As suggested at the beginning of the chapter, this limitation is due partly to the constraints placed upon attention research by the laboratory emphasis. The suggestion here is that such an approach has limited our understanding of attention due to a lack of empirical studies of attention in naturalistic, ecologically valid activities. Therefore, it is not that the model lacks empirical support but that the model predicts empirical findings from studies that have not yet been conceived due to the inferential poverty of current, implicit and explicit, models of attention.

\section{Notes}

1. I ignore the possibility of divided attention but only for the sake of brevity. One may note that in the diagram, a second aperture is larger than the other minor apertures, and one may take this to be attentive to a second domain more or less simultaneously.

2. Researchers often stress neurophysiological evidence that attention is not a unitary process. This is true at the preconscious level, which this model takes into account via the subapertures. There is no accounting here for the actual systems of attention because this is a higher level functional model rather than a lower level physiological or computational model.

3. Edelman (1987) was the first to suggest Darwinian-type competition among neuronal processes. Calvin $(1993,238)$ later posited the brain as a "Darwin machine" in which "the brain selects the sequences of schema."

4. Anthropological inaccuracies notwithstanding.

5. Again, specifying a position on divided attention is not important. It could be that two CSRs are simultaneously open halfway or are more fully open but only in succession. What matters is the proportion of their averages compared over a short period of time and that an increase of intensity in one CSR leads to a decrease in sensitivity in others.

6. Of course, the monikers postulated to indentify CSRs may be taken as more or less appropriate. While a sustenance CSR, for example, may have an emphasis on genuine nutrition at one point in time, it may have an emphasis on pleasure at another point, or any combination of the two.

7. Botvinick and Plaut state that "there are many types of routine behavior for which it is not straightforward to identify discrete, explicit goals, for example, taking a walk or playing the violin" (Botvinick and Plaut 2004, 423) and that such activities may be driven, instead, by external cues. How correct this claim is depends on what one means by "discrete, explicit goals." In the model offered here, by goals, I mean any directedness in an activity such that action in the activity can be judged in at least a tenuous way successful or not. Routine activities may be acti- 
vated by external cues, but they will still have a directedness to them, even though such directedness may be as general as preserving homeostasis, satisfying curiosity, alleviating stiffness, or killing time. Further, it should be stressed that I make no claim as to whether the goals guiding activities are conscious or to their number. It may be that a goal is unconscious while the behavior, itself, is conscious, and it may be that multiple goals within a CSR are driving an activity.

This deserves a bit more discussion. Consider John Conway's Game of Life (Gardner 1970). In this game, a simple set of instructions leads to temporally unfolding, complex, patterned movement. We can accurately say that these are parameter-constrained "movements" but not goal-driven ones. This would be equivalent to a decontextualized human action, such as raising a hand. I suggest that such actions in humans are always embedded in goal-directed activities and do not arise solely due to external cues. A domain of activity (CSR), which contains the encoding for potential actions, may arise from attentional ascendency prompted by external cues but only in conjunction with internal sensitivity, which is itself tied to some general or specific goal or goals.

8. It is true that neural network researchers often exploit nonlinear dynamics in their models of cognition (as in gradient descent learning) and that some cognitive scientists have had great success with stochastic equations that result in simulated behavior that appears to mimic features of human cognition. Nevertheless, such cognitive processes in a normal human being are still fundamentally goal driven (i.e., directed), are parameter bounded, and are related in nonrandom ways to other goal-driven, parameter-bounded cognitive processes. Thus, my claim of the syntactic nature of cognition is not damaged by the prospect of stochastic processes in cognition. If such processes occur, they likely occur at one or two stages in the cognition of attention: (1) in the online approximations of an unfolding action-as such they still occur at a level that is subservient to nonrandom, syntactic processes; (2) in the ongoing spontaneous neural activity that subtends endogenous CSR fluctuations. If both of these are the case, there would be a beautiful hierarchy of levels, with randomness embedded in syntax and syntax embedded in randomness, and so on.

9. In attention research, a cue is often understood as a preliminary stimulus that precedes a target stimulus and directs attention to it. Here, however, I am replacing the term stimulus with the term cue, to emphasize that there is no stimulus without context. Cues are primary.

10. The correctness of a formal action can be judged on either normative grounds (e.g., movements in classical ballet) or instrumental grounds (a correct golf swing, for instance, is an effective one).

11. There is only one way to swallow that I can think of, but there are an infinite number of ways to move the grape to the mouth and to chew, even if we habitually choose only one. For easy verification, observe children at the table.

\section{References}

Bindra, D. 1974. A motivational view of learning, performance, and behavior modification. Psychol. Rev. 81:199-213. 
Borghi, A. 2005. Object concepts and action. In Grounding cognition: The role of perception and action in memory, language, and thinking, ed. D. Pecher and R. A. Zwaan. Cambridge: Cambridge University Press, 8-34.

Botvinick, M., and D. C. Plaut. 2004. Doing without schema hierarchies: A recurrent connectionist approach to normal and impaired routine sequential action. Psychol. Rev. 111:395-429.

Broadbent, D. E. 1957. A mechanical model for human attention and immediate memory. Psychol. Rev. 64:205-215.

Broadbent, D. E. 1958. Perception and communication. New York: Pergamon Press.

Calvin, W. H. 1993. The unitary hypothesis: A common neural circuitry for novel manipulations, language, plan ahead, and throwing? In Tools, language, and cognition in human evolution, ed. K. R. Gibson and T. Ringold. New York: Cambridge University Press, 230-250.

Csikszentmihalyi, M. 1975. Beyond boredom and anxiety. 1st ed. San Francisco: Jossey-Bass.

Csikszentmihalyi, M. 1990. Flow: The psychology of optimal experience. 1st ed. New York: Harper $\&$ Row.

Csikszentmihalyi, M. 1997. Finding flow: The psychology of engagement with everyday life. New York: Basic Books.

Csikszentmihalyi, M., and I. S. Csikszentmihalyi. 1988. Optimal experience: Psychological studies of flow in consciousness. Cambridge: Cambridge University Press.

Edelman, G. M. 1987. Neural Darwinism: The theory of neuronal group selection. New York: Basic Books.

Faglioni, P. 1990. The frontal lobe. In Handbook of clinical and experimental neuropsychology, ed. G. Denes and L. Pizzamiglio. Hove, UK: Psychology Press, 525-569.

Gardner, M. 1970. The fantastic combinations of John Conway's new solitaire game "Life." Sci. Am. 223:120-123.

Gopnik, A., and L. Schulz. 2004. Mechanisms of theory formation in young children. Trends Cogn. Sci. 8:371-377.

Houghton, G., and T. Hartley. 1995. Parallel models of serial behaviour: Lashley revisited. Psyche $2(25)$.

Kahneman, D. 1973. Attention and effort. Englewood Cliffs, NJ: Prentice-Hall.

Mack, A., Z. Pappas, M. Silverman, and R. Gay. 2002. What we see: Inattention and the capture of attention by meaning. Conscious. Cogn. 11:488-506.

Mack, A., and I. Rock. 1998. Inattentional blindness. Cambridge: MIT Press.

Millikan, R. G. 1989. Biosemantics. J. Philos. 86:281-297.

Parasuraman, R. 1998a. The attentive brain: Issues and prospects. In The attentive brain, ed. R. Parasuraman. Cambridge: MIT Press, 3-15. 
Parasuraman, R. 1998b. The attentive brain. Cambridge: MIT Press.

Pashler, H., ed. 1998. Attention. Hove, UK: Psychology Press.

Posner, M. I. 2004. Cognitive neuroscience of attention. New York: Guilford.

Pylyshyn, Z. 1989. The role of location indexes in spatial perception: A sketch of the FINST spatial-index model. Cognition 32:65-97.

Silvia, P. J. 2006. Exploring the psychology of interest. New York: Oxford University Press.

Treisman, A. M., and G. Gelade. 1980. A feature-integration theory of attention. Cognit. Psychol. 12:97-136.

Underwood, G. 1993. The psychology of attention. New York: New York University Press. 